\title{
A GENERAL COMPARISON THEOREM FOR BACKWARD STOCHASTIC DIFFERENTIAL EQUATIONS
}

\author{
SAMUEL N. COHEN, ${ }^{* * *}$ University of Adelaide \\ ROBERT J. ELLIOTT, ${ }^{* * *}$ University of Adelaide and University of Calgary \\ CHARLES E. M. PEARCE, ${ }^{* * * * *}$ University of Adelaide
}

\begin{abstract}
A useful result when dealing with backward stochastic differential equations is the comparison theorem of Peng (1992). When the equations are not based on Brownian motion, the comparison theorem no longer holds in general. In this paper we present a condition for a comparison theorem to hold for backward stochastic differential equations based on arbitrary martingales. This theorem applies to both vector and scalar situations. Applications to the theory of nonlinear expectations are also explored.
\end{abstract}

Keywords: BSDE; comparison theorem; nonlinear expectation; dynamic risk measure

2010 Mathematics Subject Classification: Primary 60H10

Secondary 93E20

\section{Introduction}

The theory of backward stochastic differential equations (BSDEs) is an active area of research in both mathematical finance and stochastic control. Typically, we begin by defining processes $(Y, Z)$ through an equation of the form

$$
Y_{t}-\int_{(t, T]} F\left(\omega, u, Y_{u-}, Z_{u}\right) \mathrm{d} u+\int_{(t, T]} Z_{u} \mathrm{~d} M_{u}=Q .
$$

Here $Q$ is a square-integrable terminal condition, $F$ a progressively measurable 'driver' function, and $M$ an $N$-dimensional Brownian motion, all defined on a probability space with filtration generated by $M$. Recent work has also allowed the presence of jumps and the use of other underlying processes. However, these typically require the addition of another martingale process, as a martingale representation theorem may not hold. See El Karoui and Huang [13, Chapter 2] for some general results. In [5], we considered the situation where $M$ is the compensated jump martingale generated by a continuous-time, finite-state Markov chain and showed that solutions existed for equations of this type.

A fundamental result, first obtained by Peng [24], is the 'comparison theorem' for BSDEs. This result is connected to the Pontryagin maximum principle in optimal control, and, as is explored in [29, Chapter 8] for the linear case, to the theory of no-arbitrage in a financial market.

Received 20 April 2009; revision received 23 March 2010.

* Postal address: School of Mathematical Sciences, University of Adelaide, Adelaide, 5005, Australia.

** Email address: samuel.cohen@ adelaide.edu.au

*** Postal address: Haskayne School of Business, University of Calgary, Calgary, T2N 1N4, Canada.

Email address: relliott@ucalgary.ca

**** Email address: charles.pearce@ adelaide.edu.au 
In [24], the comparison theorem was established for scalar BSDEs based on $N$-dimensional Brownian motion. Various other works have extended this result, primarily through the addition of jump terms. Barles et al. [2] gave a comparison theorem for BSDEs based on $N$-dimensional Brownian motion and an independent Poisson random measure. Other particular cases, with Poisson random measures and Lévy processes, can be seen in [16], [23], [28], [29, Section 8.11], and [30]. Barles et al. [2] also included a counterexample, which shows that the conditions of the comparison theorem for simple Brownian motion are insufficient when jumps are present. In the context of continuous-time Markov chains, Cohen and Elliott [8] gave an appropriate comparison theorem. To obtain these theorems, added conditions on the driver $F$, in particular on its relation to the jump component of the underlying process, are needed. This paper generalises these conditions, by expressing them in the language of equivalent measures.

In the vector-valued context, Hu and Peng [18] considered vector-valued BSDEs based on $N$-dimensional Brownian motion, and, under a bounded stochastic viability property (or BVSP), which degenerates into the classical requirements in one dimension, presented a comparison theorem in this context. These conditions are considerably less intuitive than those in one dimension, and do not as easily lead to a theory of multidimensional nonlinear evaluations and expectations (as in [10], [28], and others). They also do not extend to the situation of BSDEs generated by processes other than Brownian motion. The conditions we present in this paper are different, and are a more natural componentwise extension of the scalar case. Various comparison theorems for vector-valued BSDEs based on continuous-time Markov chains are given in [8], however under restrictive conditions on the driver $F$. Our conditions are, in general, more difficult to verify. However, these conditions are the natural extension of the conditions derived in [6] in a discrete-time context, where it can be shown that they are the most general conditions for the componentwise monotonicity of the BSDE solutions. A future extension of this work would be to derive a BSVP for this more general class of BSDEs.

The above results are all in contexts where the simple BSDE (1), or its variants including random measures, has a solution. In these cases, an orthogonal martingale, the $\mathrm{d} L$ term in (2), below, is not required. This paper does not assume this, and the comparison theorem also applies to the generalised BSDE (2) considered in [13].

In this paper we generalise these results, by making clearer the relationship between the comparison theorem and the existence of equivalent (super)martingale measures. This highlights the relationship between the comparison theorem and no-arbitrage in a financial market, by reference to the fundamental theorem of asset pricing (see [12, Section 1.6]). By expressing the conditions for the comparison theorem in this way, the proofs become considerably simpler, and also extend naturally to BSDEs based on any martingale process, as they do not depend on a Lévy characterisation or on the Markov property.

As mentioned above, these conditions are also the natural extension of the conditions derived in [6] for discrete-time BSDEs. This fact is demonstrated in [7, Theorem 3.2]. In this discretetime framework, we can show that the requirements of the comparison theorem are the most general conditions for the monotonicity of the BSDE solutions (see [6, Theorem 7] and [7, Theorem 4.1]). Given the general result of the fundamental theorem of asset pricing, we could expect that an approach based on the existence of martingale measures is therefore an appropriate characterisation of the general requirements for the comparison theorem.

Our general approach may also have implications for considering existence and convergence results of BSDEs; however, we do not explore these here. For example, when considering BSDEs with continuous coefficients, the comparison theorem plays a fundamental role in the proof of uniqueness of solutions; see, for example, [20]. When considering convergence of 
discrete approximations of BSDEs in a non-Lipschitz setting, as in [4], the comparison theorem again is an essential result.

We demonstrate the usefulness of this result in defining dynamically consistent nonlinear expectations.

\section{BSDEs with arbitrary martingales}

Let $M$ be an arbitrary $\mathbb{R}^{N}$-valued càdlàg martingale on a filtered probability space $(\Omega, \mathcal{F}$, $\left.\left\{\mathcal{F}_{t}\right\}, \mathrm{P}\right)$ satisfying the usual assumptions of completeness and right continuity. Let $\|\cdot\|$ denote the Euclidean norm, and let $L^{0}\left(\mathcal{F}_{t}\right)$ denote the set of $\mathscr{F}_{t}$-measurable random variables $Q$ with $\mathrm{P}(\|Q\|=+\infty)=0$.

For a fixed deterministic $T \leq+\infty$ and a given integrable, progressively measurable function $F: \Omega \times[0, T] \times \mathbb{R}^{K} \times \mathbb{R}^{K \times N} \rightarrow \mathbb{R}^{K}$, we shall consider equations of the form

$$
Y_{r}-\int_{(r, t]} F\left(\omega, u, Y_{u-}, Z_{u}\right) \mathrm{d} u+\int_{(r, t]} Z_{u} \mathrm{~d} M_{u}+\int_{(r, t]} \mathrm{d} L_{u}=Q,
$$

where $0 \leq r \leq t \leq T$ and $Q$ is an $\mathbb{R}^{K}$-valued $\mathcal{F}_{t}$-measurable terminal condition. A solution to (2) is a triple $(Y, \bar{Z}, L)$, with $Y$ a càdlàg, adapted, $\mathbb{R}^{K}$-valued process, $Z$ a predictable $\mathbb{R}^{K \times N_{-}}$ valued process and $L$ an $\mathbb{R}^{K}$-valued càdlàg martingale, with $L_{0}=0$, which is orthogonal to $M$, that is, the $(K \times N)$-dimensional matrix process

$$
\langle L, M\rangle=\left(\left\langle e_{i}^{\top} L, e_{j}^{\top} M\right\rangle\right)_{i=1, \ldots, K}^{j=1, \ldots, N} \equiv 0 .
$$

Here $e_{i}$ is the $i$ th standard basis vector in $\mathbb{R}^{N}$ (or $\mathbb{R}^{K}$, as appropriate). We here restrict our attention to deterministic $T$; however, through appropriate modification of the driver $F$, it is easy to see that this extends to the case when $T$ is a stopping time (see [8]). We know from [13] that, under certain assumptions about $M, F$, and $Q$, this equation will always have a solution $(Y, Z, L)$. If we consider a space such that a martingale representation theorem holds for $M$, or we allow $M$ to become infinite-dimensional, as in [9], then, without loss of generality, $L=0$. We assume here that the driver $F$ is integrated with respect to time $(\mathrm{d} u)$; however, the general case, considered in [13], where $\mathrm{d} u$ is replaced with $\mathrm{d} C_{u}$ for $C$ a continuous, adapted, increasing process, is a straightforward modification of the results given here. (The only slight difficulty is in obtaining an appropriate version of Grönwall's inequality.)

As the focus of this paper is on comparison results, rather than proving the existence of BSDE solutions, we shall refrain from explicitly making the assumptions of [13] or [9], and, in general, denote by $\mathbb{Q}_{s, t}^{F} \subseteq L^{0}\left(\mathcal{F}_{t}\right)$ the set of values $Q$ such that, for all $r \in[s, t],(2)$ has a unique solution, up to indistinguishability on $[s, t] \times \Omega$ for the triple $\left(Y_{r}, \int_{(s, r]} Z_{u} \mathrm{~d} M_{u}, L_{r}\right)$.

This solution may be constrained to satisfy certain conditions, for example, in [5] and [13] it is required that $\mathrm{E}\left[\sup _{r \in[s, t]}\left\|Y_{r}\right\|^{2} \mid \mathcal{F}_{s}\right]<+\infty$. In this case, the solution may only be unique among those processes satisfying these constraints. We shall make the very weak assumption that, for $r \in[s, t]$, this solution satisfies the integrability assumption

$$
\mathrm{E}\left[\left\|Y_{r}\right\| \mid \mathcal{F}_{S}\right]<+\infty \quad \text { P-almost surely (P-a.s.). }
$$

It is clear that this assumption will not, in general, be sufficient for a unique solution to exist; however, it is sufficient to derive the comparison properties of interest here. We shall take any other conditions necessary for uniqueness (for example, square-integrability of the solution) as implicit. 
Lemma 1. The following properties of $\mathbb{Q}_{s, t}^{F}$ are immediately apparent.

1. For all $s \leq r \leq t, \mathbb{Q}_{s, t}^{F} \subseteq \mathbb{Q}_{r, t}^{F}$.

2. For all $t \leq T$, without loss of generality, $\mathbb{Q}_{t, t}^{F}=L^{0}\left(\mathcal{F}_{t}\right)$.

3. For $s \leq t$, let $Q \in \mathbb{Q}_{s, t}^{F}$ and let $Y_{r}$ satisfy (2). Then, for all $r \in[s, t], Y_{r} \in \mathbb{Q}_{s, r}^{F}$.

4. $\mathbb{Q}_{s, t}^{F} \subseteq\left\{Q \in L^{0}\left(\mathcal{F}_{t}\right): \mathrm{E}\left[\|Q\| \mid \mathcal{F}_{s}\right]<+\infty\right\}$.

Proof. Property 1 follows from the fact that, if we have a unique solution to (2) on $[s, t]$ then we have a unique solution on $[r, t] \subseteq[s, t]$. Property 2 is because (2) degenerates into the tautology $Y_{t}=Q$, which clearly has a unique solution up to indistinguishability. Property 3 is simply due to a rearrangement of (2). Property 4 is due to the assumed integrability condition, evaluated for $Y_{t}=Q$.

\section{Comparison properties}

We now seek to derive conditions, similar to those in [24], such that a comparison property holds with regard to BSDE solutions. For the sake of generality, we shall state these in a form such that the conditions may depend on the solutions $(Y, Z, L)$. We also allow our solution processes to be multidimensional. In practice, the conditions imposed may be difficult to verify, particularly in the multidimensional case; however, they allow us to unite, as special cases, the various comparison results which have previously been obtained. We shall present, in Section 4, examples of BSDEs where these conditions are satisfied for all solutions $(Y, Z, L)$.

In the following, a vector inequality is assumed to hold componentwise.

Theorem 1. (Comparison theorem.) Suppose that we have two BSDEs corresponding to coefficients and terminal values $\left(F^{1}, Q^{1}\right)$ and $\left(F^{2}, Q^{2}\right), Q^{1} \in \mathbb{Q}_{s, t}^{F^{1}}, Q^{2} \in \mathbb{Q}_{s, t}^{F^{2}}$. Let $\left(Y^{1}, Z^{1}, L^{1}\right)$ and $\left(Y^{2}, Z^{2}, L^{2}\right)$ be the associated solutions. We suppose that the following conditions hold.

(i) $Q^{1} \geq Q^{2}$, P-a.s.

(ii) $(\mathrm{d} u \times \mathrm{P})$-a.s. on $[s, t] \times \Omega, F^{1}\left(\omega, u, Y_{u-}^{2}, Z_{u}^{2}\right) \geq F^{2}\left(\omega, u, Y_{u-}^{2}, Z_{u}^{2}\right)$.

(iii) For each $i$, there exists a measure $\tilde{\mathrm{P}}_{i}$ equivalent to $\mathrm{P}$ such that the ith component of $X$, as defined for $r \in[s, t]$ by

$$
\begin{aligned}
e_{i}^{\top} X_{r}:= & -\int_{(s, r]} e_{i}^{\top}\left[F^{1}\left(\omega, u, Y_{u-}^{2}, Z_{u}^{1}\right)-F^{1}\left(\omega, u, Y_{u-}^{2}, Z_{u}^{2}\right)\right] \mathrm{d} u \\
& +\int_{(s, r]} e_{i}^{\top}\left[Z_{u}^{1}-Z_{u}^{2}\right] \mathrm{d} M_{u}+e_{i}^{\top}\left[L_{r}^{1}-L_{r}^{2}\right]
\end{aligned}
$$

is a $\tilde{\mathrm{P}}_{i}$-supermartingale on $[s, t]$.

(iv) If, for all $r \in[s, t]$,

$$
\begin{aligned}
& e_{i}^{\top} Y_{r}^{1}-\mathrm{E}_{\tilde{\mathrm{P}}_{i}}\left[\int_{(r, t]} e_{i}^{\top} F^{1}\left(\omega, u, Y_{u-}^{1}, Z_{u}^{1}\right) \mathrm{d} u \mid \mathcal{F}_{r}\right] \\
& \quad \geq e_{i}^{\top} Y_{r}^{2}-\mathrm{E}_{\tilde{\mathrm{P}}_{i}}\left[\int_{(r, t]} e_{i}^{\top} F^{1}\left(\omega, u, Y_{u-}^{2}, Z_{u}^{1}\right) \mathrm{d} u \mid \mathcal{F}_{r}\right],
\end{aligned}
$$

for all $i$, then $Y_{r}^{1} \geq Y_{r}^{2}$ for all $r \in[s, t]$ componentwise.

It is then true that $Y^{1} \geq Y^{2}$ on $[s, t]$, except possibly on some evanescent set. 
Proof. We omit the $\omega$ and $u$ arguments of $F$ for clarity. Then, for $r \in[s, t]$,

$$
\begin{aligned}
& Y_{r}^{1}-Y_{r}^{2}-\int_{(r, t]}\left[F^{1}\left(Y_{u-}^{1}, Z_{u}^{1}\right)-F^{2}\left(Y_{u-}^{2}, Z_{u}^{2}\right)\right] \mathrm{d} u \\
& +\int_{(r, t]}\left[Z_{u}^{1}-Z_{u}^{2}\right] \mathrm{d} M_{u}+\int_{(r, t]} \mathrm{d} L_{u}^{1}-\int_{(t, T]} \mathrm{d} L_{u}^{2} \\
& \quad=Q^{1}-Q^{2} \\
& \quad \geq 0,
\end{aligned}
$$

which can be rearranged to give

$$
\begin{aligned}
Y_{r}^{1}- & Y_{r}^{2}-\int_{(r, t]}\left[F^{1}\left(Y_{u-}^{1}, Z_{u}^{1}\right)-F^{1}\left(Y_{u-}^{2}, Z_{u}^{1}\right)\right] \mathrm{d} u \\
\geq & \int_{(r, t]}\left[F^{1}\left(Y_{u-}^{2}, Z_{u}^{2}\right)-F^{2}\left(Y_{u-}^{2}, Z_{u}^{2}\right)\right] \mathrm{d} u+\int_{(r, t]}\left[F^{1}\left(Y_{u-}^{2}, Z_{u}^{1}\right)-F^{1}\left(Y_{u-}^{2}, Z_{u}^{2}\right)\right] \mathrm{d} u \\
& -\int_{(r, t]}\left[Z_{u}^{1}-Z_{u}^{2}\right] \mathrm{d} M_{u}-\int_{(r, t]} \mathrm{d} L_{u}^{1}+\int_{(r, t]} \mathrm{d} L_{u}^{2} .
\end{aligned}
$$

We have $\int_{(r, t]}\left[F^{1}\left(Y_{u-}^{2}, Z_{u}^{2}\right)-F^{2}\left(Y_{u-}^{2}, Z_{u}^{2}\right)\right] \mathrm{d} u \geq 0$ by assumption (ii). As $e_{i}^{\top} X_{r}$ is a $\tilde{\mathrm{P}}_{i^{-}}$ supermartingale, we know that the process given by

$$
\begin{aligned}
e_{i}^{\top} \tilde{X}_{r}:= & e_{i}^{\top} X_{r}-\mathrm{E}_{\tilde{\mathrm{P}}_{i}}\left[e_{i}^{\top} X_{t} \mid \mathcal{F}_{r}\right] \\
= & \mathrm{E}_{\tilde{\mathrm{P}}_{i}}\left[\int_{(r, t]} e_{i}^{\top}\left[F^{1}\left(Y_{u-}^{2}, Z_{u}^{1}\right)-F^{1}\left(Y_{u-}^{2}, Z_{u}^{2}\right)\right] \mathrm{d} u\right. \\
& \left.-\int_{(r, t]} e_{i}^{\top}\left[Z_{u}^{1}-Z_{u}^{2}\right] \mathrm{d} M_{u}-\int_{(r, t]} e_{i}^{\top} \mathrm{d} L_{u}^{1}+\int_{(r, t]} e_{i}^{\top} d L_{u}^{2} \mid \mathcal{F}_{r}\right]
\end{aligned}
$$

is also a $\tilde{\mathrm{P}}_{i}$-supermartingale, with $e_{i}^{\top} \tilde{X}_{t}=0, \tilde{\mathrm{P}}_{i}$-a.s. Hence, $e_{i}^{\top} \tilde{X}_{r} \geq 0$.

For each $i$, taking a $\tilde{\mathrm{P}}_{i} \mid \mathcal{F}_{r}$ conditional expectation throughout (3) and premultiplying by $e_{i}^{\top}$ gives

$$
e_{i}^{\top} Y_{r}^{1}-e_{i}^{\top} Y_{r}^{2}-\mathrm{E}_{\tilde{\mathrm{P}}_{i}}\left[\int_{(r, t]} e_{i}^{\top}\left[F^{1}\left(Y_{u-}^{1}, Z_{u}^{1}\right)-F^{1}\left(Y_{u-}^{2}, Z_{u}^{1}\right)\right] \mathrm{d} u \mid \mathcal{F}_{r}\right] \geq 0 .
$$

By assumption (iv), this then proves that $Y_{r}^{1} \geq Y_{r}^{2}$ componentwise P-a.s. for each $r \in[s, t]$. As $Y^{1}-Y^{2}$ is càdlàg, $Y^{1}-Y^{2}$ is indistinguishable from a nonnegative process and, therefore, the inequality holds up to evanescence.

Corollary 1. Theorem 1 remains true if assumption (iii) and assumption (iv) are replaced by the following.

(iii') For each $i$, there exists a measure $\tilde{\mathrm{P}}_{i}$ equivalent to $\mathrm{P}$ such that the ith component of $X$, as defined for $r \in[s, t]$

$$
\begin{aligned}
e_{i}^{\top} X_{r}:= & -\int_{(s, r]} e_{i}^{\top}\left[F^{1}\left(\omega, u, Y_{u-}^{1}, Z_{u}^{1}\right)-F^{1}\left(\omega, u, Y_{u-}^{1}, Z_{u}^{2}\right)\right] \mathrm{d} u \\
& +\int_{(s, r]} e_{i}^{\top}\left[Z_{u}^{1}-Z_{u}^{2}\right] \mathrm{d} M_{u}+e_{i}^{\top}\left[L_{t}^{1}-L_{t}^{2}\right],
\end{aligned}
$$

is a $\tilde{\mathrm{P}}_{i}$-supermartingale on $[s, t]$. 


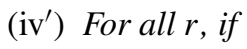

$$
\begin{aligned}
& e_{i}^{\top} Y_{r}^{1}-\mathrm{E}_{\tilde{\mathrm{P}}_{i}}\left[\int_{(r, t]} e_{i}^{\top} F^{1}\left(\omega, u, Y_{u-}^{1}, Z_{u}^{2}\right) \mathrm{d} u \mid \mathcal{F}_{r}\right] \\
& \quad \geq e_{i}^{\top} Y_{r}^{2}-\mathrm{E}_{\tilde{\mathrm{P}}_{i}}\left[\int_{(r, t]} e_{i}^{\top} F^{1}\left(\omega, u, Y_{u-}^{2}, Z_{u}^{2}\right) \mathrm{d} u \mid \mathcal{F}_{r}\right]
\end{aligned}
$$

for all $i$ then $Y_{r}^{1} \geq Y_{r}^{2}$ componentwise.

Proof. In this case, the decomposition in (3) and the definition of $\tilde{X}$ in (4) are correspondingly changed. The rest of the proof remains valid.

Remark 1. In the scalar case $(K=1)$ when $M$ is a Brownian motion generating $\{\mathcal{F}\}$ and $F^{1}\left(\omega, u, Y_{u-}, \cdot\right)$ is uniformly Lipschitz continuous in $Z_{u}$, and in many other cases, we can use the standard results of Girsanov's theorem to show that Theorem 1(iii) holds.

Remark 2. A significant special case, particularly in the context of dynamic risk measures, is when $F$ is assumed not to depend on $Y$. (See, for example, [3], [6], [8], [27], and [28].) In this case, Theorem 1(iv) is trivial.

The following backwards version of Grönwall's inequality will be useful.

Lemma 2. Suppose that $\phi:[s, t] \rightarrow \mathbb{R}$ is such that, for constants $\alpha \geq 0$ and $\beta \geq 0$,

$$
\phi_{r} \leq \alpha+\beta \int_{(r, t]} \phi_{u} \mathrm{~d} u
$$

for all $r \in[s, t]$. Then $\phi_{r} \leq \alpha \mathrm{e}^{\beta(t-r)}$.

Proof. Write $\eta_{r}=\alpha+\beta \int_{(r, t]} \phi_{u} \mathrm{~d} u$. Then

$$
\frac{\mathrm{d} \eta_{r}}{\mathrm{~d} r}=-\beta \phi_{r} \geq-\beta \eta_{r} .
$$

Hence, if $v_{r}=e^{\beta r} \eta_{r}$,

$$
\frac{\mathrm{d} v_{r}}{\mathrm{~d} r}=\beta \mathrm{e}^{\beta r} \eta_{r}+\mathrm{e}^{\beta r} \frac{\mathrm{d} \eta_{r}}{\mathrm{~d} r} \geq 0 .
$$

This implies that $v$ is nondecreasing, and so $v_{r} \leq v_{t}$, which, by rearrangement, gives

$$
\eta_{r} \leq \alpha \mathrm{e}^{\beta(t-r)} .
$$

Finally, as $\phi_{r} \leq \eta_{r}$, we have the result.

Theorem 2. Consider the scalar $(K=1)$ case, where, for all $u \in[s, t], F^{1}\left(\omega, u, \cdot, Z_{u}^{1}\right)$ is uniformly Lipschitz continuous with respect to $Y$, that is, there exists $c \geq 0$ such that, for any $Y^{1}$ and $Y^{2}$,

$$
\left|F^{1}\left(\omega, u, Y_{u-}^{1}, Z_{u}^{1}\right)-F^{1}\left(\omega, u, Y_{u-}^{2}, Z_{u}^{1}\right)\right| \leq c\left|Y_{u-}^{1}-Y_{u-}^{2}\right|, \quad(\mathrm{d} u \times \mathrm{P})-\text { a.s. }
$$

Then assumption (iv) of Theorem 1 can be omitted. 
Proof. As we are in the scalar case, we can omit the $e_{i}$ from the statement of the assumption. Hence, we wish to show that, given, for all $r \in[s, t]$,

$$
\begin{aligned}
Y_{r}^{1}- & \mathrm{E}_{\tilde{\mathrm{P}}}\left[\int_{(r, t]} F^{1}\left(\omega, u, Y_{u-}^{1}, Z_{u}^{1}\right) \mathrm{d} u \mid \mathcal{F}_{r}\right] \\
& \geq Y_{r}^{2}-\mathrm{E}_{\tilde{\mathrm{P}}}\left[\int_{(r, t]} F^{1}\left(\omega, u, Y_{u-}^{2}, Z_{u}^{1}\right) \mathrm{d} u \mid \mathcal{F}_{r}\right],
\end{aligned}
$$

we must have $Y_{r}^{1} \geq Y_{r}^{2}$. For simplicity, let $\delta Y:=Y^{1}-Y^{2}$.

It is clear from the problem and the recursivity of BSDE solutions that we can replace $t$ with any stopping time $\tau \leq t$ such that $\delta Y_{\tau} \geq 0$.

Suppose that on some nonnull set $A \in \mathcal{F}, \delta Y_{u}<0$ for some $u \in[s, t]$. As $\delta Y$ is adapted and right continuous, this implies that there are stopping times $\sigma$ and $\tau$ such that $\delta Y_{u}<0$ for all $u \in[\sigma, \tau)$, and $\sigma<\tau$ on $A$. Without loss of generality, let $\tau$ be the largest such upper bound. Then, as $\delta Y_{t} \geq 0$ and $\tau \leq t$, it follows that $\delta Y_{\tau} \geq 0$. Let $I_{A}$ denote the indicator function of a set. Replacing $t$ with $\tau$ in the above inequality, we know that

$$
\begin{aligned}
\mathrm{E}_{\tilde{\mathrm{P}}}\left[I_{r \in[\sigma, \tau)}\left|\delta Y_{r}\right|\right] & =\mathrm{E}_{\tilde{\mathrm{P}}}\left[-I_{r \in[\sigma, \tau)} \delta Y_{r}\right] \\
& \leq \mathrm{E}_{\tilde{\mathrm{P}}}\left[-I_{r \in[\sigma, \tau)} \int_{(r, \tau]}\left(F^{1}\left(\omega, u, Y_{u-}^{1}, Z_{u}^{1}\right)-F^{1}\left(\omega, u, Y_{u-}^{2}, Z_{u}^{1}\right)\right) \mathrm{d} u\right] \\
& \leq \mathrm{E}_{\tilde{\mathrm{P}}}\left[I_{r \in[\sigma, \tau)} \int_{(r, \tau]} c\left|\delta Y_{u-}\right| \mathrm{d} u\right] \\
& \leq c \int_{(r, t]} \mathrm{E}_{\tilde{\mathrm{P}}}\left[I_{u \in[\sigma, \tau)}\left|\delta Y_{u-}\right|\right] \mathrm{d} u .
\end{aligned}
$$

As $\delta Y_{u}$ is càdlàg, we can omit the left limit inside the integral, and an application of Lemma 2 implies that $\mathrm{E}_{\tilde{\mathrm{P}}}\left[I_{r \in[\sigma, \tau[}\left|\delta Y_{r}\right|\right] \leq 0$, that is, $\sigma=\tau$ a.s., and so $A$ is a null set. By contradiction, we see that $\delta Y_{u} \geq 0$ for all $u \in[s, t]$.

Remark 3. By Theorem 2 and Remark 1, we can see that the classical inequality of Peng [24] is simply a special case of Theorem 1. Similarly, the scalar comparison in [8, Theorem 4.2] also follows as a special case. However, as shown in the counterexamples presented in [8, Example 5.1] for the vector case, Theorem 1(iv) remains nontrivial (cf. Remark 5 below).

Definition 1. The comparison between $Y^{1}$ and $Y^{2}$ will be called strict on $[s, t]$ if the conditions of Theorem 1 hold, and if, for any $A \in \mathcal{F}_{s}$ such that $Y_{s}^{1}=Y_{s}^{2}$, P-a.s. on $A$, we have $Y_{u}^{1}=Y_{u}^{2}$ on $[s, t] \times A$, up to evanescence.

Lemma 3. If the comparison is strict on $[s, t]$ then, for any $A \in \mathcal{F}_{s}$ such that $Y_{s}^{1}=Y_{s}^{2}$, P-a.s. on $A$, it follows that

- $Q^{1}=Q^{1}$, P-a.s. on $A$,

- $F^{1}\left(\omega, u, Y_{u-}^{2} Z_{u}^{2}\right)=F^{2}\left(\omega, u, Y_{u-}^{2}, Z_{u}^{2}\right),(\mathrm{d} u \times \mathrm{P})$-a.s. on $[s, t] \times A$, and

- for $r \in[s, t]$, up to indistinguishability, on $A$,

$$
\int_{(s, r]} Z_{u}^{1} \mathrm{~d} M_{u}=\int_{(s, r]} Z_{u}^{2} \mathrm{~d} M_{u}
$$

and

$$
L_{r}^{1}=L_{r}^{2}
$$


Proof. We omit the $\omega$ and $t$ arguments of $F^{1}$ and $F^{2}$ for clarity. Let $\tilde{X}$ be as in (4), and let $S$ be the process defined by

$$
\begin{aligned}
e_{i}^{\top} S_{r}:= & e_{i}^{\top} \mathrm{E}_{\tilde{\mathrm{P}}_{i}}\left[Q^{1}-Q^{2} \mid \mathcal{F}_{r}\right] \\
& +e_{i}^{\top} \mathrm{E}_{\tilde{\mathrm{P}}_{i}}\left[\int_{(r, t]}\left[F^{1}\left(Y_{u-}^{2}, Z_{u}^{2}\right)-F^{2}\left(Y_{u-}^{2}, Z_{u}^{2}\right)\right] \mathrm{d} u \mid \mathcal{F}_{r}\right]+e_{i}^{\top} \tilde{X}_{r} .
\end{aligned}
$$

Then $e_{i}^{\top} S$ is a $\tilde{\mathrm{P}}_{i}$-supermartingale, as the first term is a $\tilde{\mathrm{P}}_{i}$-martingale, the second is nonincreasing in $r$ by assumption (ii) of Theorem 1 , and the third is a $\tilde{\mathrm{P}}_{i}$-supermartingale by assumption (iii) of Theorem 1. Furthermore, each of these terms is nonnegative.

Taking a $\tilde{\mathrm{P}} \mid \mathcal{F}_{r}$ conditional expectation through (2), we have, for all $r \in[s, t]$,

$$
Y_{r}^{1}-Y_{r}^{2}=S_{r}+\mathrm{E}_{\tilde{\mathrm{P}}}\left[\int_{(r, t]}\left[F^{1}\left(Y_{u-}^{1}, Z_{u}^{1}\right)-F^{1}\left(Y_{u-}^{2}, Z_{u}^{1}\right)\right] \mathrm{d} u \mid \mathcal{F}_{r}\right] .
$$

If $Y_{r}^{1}=Y_{r}^{2}$ on $[s, t] \times A$ up to evanescence then it is clear from (6) that $S_{r}=0, \mathrm{P}$-a.s. on $[s, t] \times A$. Hence, by nonnegativity, each of the terms on the right-hand side of (5) must be 0 . The first two points of the lemma immediately follow.

Consider the BSDE (2) satisfied by $Y^{2}$. As $F^{1}\left(Y_{u-}^{2}, Z_{u}^{2}\right)=F^{2}\left(Y_{u-}^{2}, Z_{u}^{2}\right),(\mathrm{d} u \times \mathrm{P})$-a.s. on $[s, t] \times A$ and $Q^{1}=Q^{2}$, P-a.s. on $A$, we know that

$$
Y_{r}^{2}-\int_{(r, t]} F^{2}\left(Y_{u-}^{2}, Z_{u}^{2}\right) \mathrm{d} u+\int_{(r, t]} Z_{u}^{2} \mathrm{~d} M_{u}+\int_{(r, t]} \mathrm{d} L_{u}^{2}=Q^{2}
$$

is P-a.s. equal to

$$
Y_{r}^{2}-\int_{(r, t]} F^{1}\left(Y_{u-}^{2}, Z_{u}^{2}\right) \mathrm{d} u+\int_{(r, t]} Z_{u}^{2} \mathrm{~d} M_{u}+\int_{(r, t]} \mathrm{d} L_{u}^{2}=Q^{1} .
$$

Hence, in $A,\left(Y^{2}, Z^{2}, L^{2}\right)$ is a solution at time $r$ to the BSDE defining the triple $\left(Y^{1}, Z^{1}, L^{1}\right)$.

By assumption, as $Q^{1} \in \mathbb{Q}_{s, t}^{F^{1}}$, the solution to this BSDE is unique up to indistinguishability for $\left(Y, \int_{(s, \cdot]} Z_{u} \mathrm{~d} M_{u}, L\right)$ on $[s, t] \times \Omega$. It follows that, for each $r,\left(Y_{r}^{1}, \int_{(s, r]} Z_{u}^{1} \mathrm{~d} M_{u}, L_{r}^{1}\right)$ is unique up to equality P-a.s., and, therefore, $\int_{(s, r]} Z_{u}^{1} \mathrm{~d} M_{u}=\int_{(s, r]} Z_{u}^{2} \mathrm{~d} M_{u}$ and $L_{r}^{1}=L_{r}^{2}$, P-a.s. on $A$. As all of these processes are càdlàg, it follows from [14, Lemma 2.21] that they are indistinguishable on $[s, t] \times A$.

Theorem 3. (Strict comparison 1.) Consider the scalar $(K=1)$ case, where $F^{1}$ is such that Theorems 1 and 2 hold. Then the comparison is strict on $[s, t]$.

Proof. Again, as $K=1$, we can omit $e_{i}$ from all equations, and we omit the $\omega$ and $t$ arguments of $F^{1}$ and $F^{2}$ for clarity. Let $S_{r}$ be as defined in (5), and note that $S$ is a nonnegative $\tilde{\mathrm{P}}$-supermartingale.

Taking a $\tilde{\mathrm{P}} \mid \mathcal{F}_{S}$ conditional expectation of (6) gives

$$
\begin{aligned}
\mathrm{E}_{\tilde{\mathrm{P}}}\left[Y_{r}^{1}-Y_{r}^{2} \mid \mathcal{F}_{s}\right]= & \mathrm{E}_{\tilde{\mathrm{P}}}\left[S_{r}+\int_{(s, t]}\left[F^{1}\left(Y_{u-}^{1}, Z_{u}^{1}\right)-F^{1}\left(Y_{u-}^{2}, Z_{u}^{1}\right)\right] \mathrm{d} u \mid \mathcal{F}_{s}\right] \\
& -\mathrm{E}_{\tilde{\mathrm{P}}}\left[\int_{(s, r]}\left[F^{1}\left(Y_{u-}^{1}, Z_{u}^{1}\right)-F^{1}\left(Y_{u-}^{2}, Z_{u}^{1}\right)\right] \mathrm{d} u \mid \mathcal{F}_{s}\right] \\
\leq & S_{s}+\mathrm{E}_{\tilde{\mathrm{P}}}\left[\int_{(s, t]}\left[F^{1}\left(Y_{u-}^{1}, Z_{u}^{1}\right)-F^{1}\left(Y_{u-}^{2}, Z_{u}^{1}\right)\right] \mathrm{d} u \mid \mathcal{F}_{s}\right] \\
& +\int_{(s, r]} \mathrm{E}_{\tilde{\mathrm{P}}}\left[\left|F^{1}\left(Y_{u-}^{1}, Z_{u}^{1}\right)-F^{1}\left(Y_{u-}^{2}, Z_{u}^{1}\right)\right| \mid \mathcal{F}_{s}\right] \mathrm{d} u
\end{aligned}
$$




$$
\begin{aligned}
\leq & S_{s}+\mathrm{E}_{\tilde{\mathrm{P}}}\left[\int_{(s, t]}\left[F^{1}\left(Y_{u-}^{1}, Z_{u}^{1}\right)-F^{1}\left(Y_{u-}^{2}, Z_{u}^{1}\right)\right] \mathrm{d} u \mid \mathcal{F}_{s}\right] \\
& +c \int_{(s, r]} \mathrm{E}_{\tilde{\mathrm{P}}}\left[\left|Y_{u-}^{1}-Y_{u-}^{2}\right| \mid \mathcal{F}_{s}\right] \mathrm{d} u .
\end{aligned}
$$

We know, from (6) and the assumption that $Y_{s}^{1}-Y_{s}^{2}=0$ on $A \in \mathcal{F}_{s}$, that

$$
I_{A} S_{s}+I_{A} \mathrm{E}_{\tilde{\mathrm{P}}}\left[\int_{(s, t]}\left[F^{1}\left(Y_{u-}^{1}, Z_{u}^{1}\right)-F^{1}\left(Y_{u-}^{2}, Z_{u}^{1}\right)\right] \mathrm{d} u \mid \mathcal{F}_{s}\right]=I_{A}\left(Y_{s}^{1}-Y_{s}^{2}\right)=0,
$$

and so, as $Y^{1}-Y^{2}$ is nonnegative by Theorem 1, premultiplication of (7) by $I_{A}$ and taking an expectation gives

$$
\mathrm{E}_{\tilde{\mathrm{P}}}\left[I_{A}\left(Y_{r}^{1}-Y_{r}^{2}\right)\right] \leq c \int_{(s, r]} \mathrm{E}_{\tilde{\mathrm{P}}}\left[I_{A}\left(Y_{u-}^{1}-Y_{u-}^{2}\right)\right] \mathrm{d} u .
$$

An application of (the forward version of) Grönwall's lemma then yields

$$
\mathrm{E}_{\tilde{\mathrm{P}}}\left[I_{A}\left(Y_{r}^{1}-Y_{r}^{2}\right)\right] \leq 0,
$$

which, by nonnegativity, implies that $Y_{r}^{1}=Y_{r}^{2}, \tilde{\mathrm{P}}$-a.s. on $A$. Again, as $Y^{1}-Y^{2}$ is càdlàg, this shows that $Y^{1}=Y^{2}$ on $[s, t] \times A$, up to evanescence.

Remark 4. Theorems 2, 3, and 4 can also be modified in the same way as in Corollary 1; in this case the assumptions of the theorems will refer to $F^{1}\left(\omega, u, \cdot, Z_{u}^{2}\right)$.

Remark 5. In a vector setting, it is easy to see that, if the $i$ th component of $F^{1}$ depends only on the $i$ th component of $Y$, that is, we can write

$$
e_{i}^{\top} F^{1}\left(\omega, t, Y_{t-}, Z_{t}\right)=F_{i}\left(\omega, t, e_{i}^{\top} Y_{t-}, Z_{t}\right)
$$

for some $F_{i}$, and if $F_{i}$ is uniformly Lipschitz in $e_{i}^{\top} Y_{t-}$, then the proofs of Theorems 2 and 3 can be extended to cover these cases, simply by considering each component separately.

In this case, the strict comparison will apply componentwise, that is, if, for some $A \in \mathcal{F}_{s}$, we have $e_{i}^{\top} Y_{s}^{1}=e_{i}^{\top} Y_{s}^{2}$, then $e_{i}^{\top} Y_{u}^{1}=e_{i}^{\top} Y_{u}^{2}$ on $[s, t] \times A$, up to evanescence.

If $F^{1}$ does not depend on $Y_{t-}$ then this is clearly the case (as $F^{1}$ is uniformly Lipschitz with constant $c=0$ ).

In some situations, particularly in the vector context, this result may be insufficient. The following theorem addresses some such cases.

Theorem 4. (Strict comparison 2.) Suppose that we have two BSDEs satisfying the conditions of Theorem 1. Suppose furthermore that Theorem 1(iv) is satisfied in such a way that, for all $A \in \mathcal{F}_{s}$,

$$
\begin{aligned}
e_{i}^{\top} Y_{r}^{1}-\mathrm{E}_{\tilde{\mathrm{P}}_{i}}\left[\int_{(r, t]} e_{i}^{\top} F^{1}\left(\omega, u, Y_{u-}^{1}, Z_{u}^{1}\right) \mathrm{d} u \mid \mathcal{F}_{r}\right] \\
=e_{i}^{\top} Y_{r}^{2}-\mathrm{E}_{\tilde{\mathrm{P}}_{i}}\left[\int_{(r, t]} e_{i}^{\top} F^{1}\left(\omega, u, Y_{u-}^{2}, Z_{u}^{1}\right) \mathrm{d} u \mid \mathcal{F}_{r}\right]
\end{aligned}
$$

P-a.s. on A for all $i$ and all $r \in[s, t]$, if and only if $Y_{r}^{1}=Y_{r}^{2}$, P-a.s. on A for all $r \in[s, t]$. Then the comparison is strict on $[s, t]$. 
Proof. As in Theorem 1, the weak comparison holds, that is, $Y_{r}^{1} \geq Y_{r}^{2}$, P-a.s. on $[s, t]$. Recall that the measures $\mathrm{P}$ and $\tilde{\mathrm{P}}_{i}$ were assumed to be equivalent, and, hence, any statement up to equality $\tilde{\mathrm{P}}_{i}$-a.s. could equivalently be made $\mathrm{P}$-a.s.

As before, $e_{i}^{\top} \tilde{X}$, defined in (4), is a $\tilde{\mathrm{P}}_{i}$-supermartingale with $e_{i}^{\top} \tilde{X}_{t}=0$. We know that $Y_{s}^{1}=Y_{s}^{2}$ on $A$, and, hence, by (8),

$$
Y_{s}^{1}-Y_{s}^{2}-\mathrm{E}_{\tilde{\mathrm{P}}}\left[\int_{(s, t]}\left[F^{1}\left(Y_{u-}^{1}, Z_{u}^{1}\right)-F^{1}\left(Y_{u-}^{2}, Z_{u}^{1}\right)\right] \mathrm{d} u \mid \mathcal{F}_{s}\right]=0 .
$$

As in (3), this implies that

$$
\begin{aligned}
0 \geq \mathrm{E}_{\tilde{\mathrm{P}}_{i}}\left[\int_{(s, t]}\left[F^{1}\left(Y_{u-}^{2}, Z_{u}^{2}\right)-F^{2}\left(Y_{u-}^{2}, Z_{u}^{2}\right)\right] \mathrm{d} u \mid \mathcal{F}_{s}\right] \\
+\mathrm{E}_{\tilde{\mathrm{P}}_{i}}\left[\int_{(s, t]}\left[F^{1}\left(Y_{u-}^{2}, Z_{u}^{1}\right)-F^{1}\left(Y_{u-}^{2}, Z_{u}^{2}\right)\right] \mathrm{d} u\right. \\
\left.\quad-\int_{(s, t]}\left[Z_{u}^{1}-Z_{u}^{2}\right]^{\top} \mathrm{d} M_{u}-\int_{(s, t]}\left(\mathrm{d} L_{u}^{1}-\mathrm{d} L_{u}^{2}\right) \mid \mathcal{F}_{s}\right] .
\end{aligned}
$$

Premultiplying by $e_{i}^{\top}$, we have

$$
0 \geq \mathrm{E}_{\tilde{\mathrm{P}}_{i}}\left[\int_{(s, t]} e_{i}^{\top}\left[F^{1}\left(Y_{u-}^{2}, Z_{u}^{2}\right)-F^{2}\left(Y_{u-}^{2}, Z_{u}^{2}\right)\right] \mathrm{d} u \mid \mathcal{F}_{s}\right]+e_{i}^{\top} \tilde{X}_{s} .
$$

Both of the terms on the right-hand side are nonnegative, and, hence, both must be 0 on $A$.

If $e_{i}^{\top} \tilde{X}_{s}=0$ on $A$ then $e_{i}^{\top} \tilde{X}_{r}=0$ on $A$ for all $r \geq s$, as $e_{i}^{\top} \tilde{X}$ is a nonnegative $\tilde{\mathrm{P}}_{i}$-supermartingale. Similarly,

$$
\mathrm{E}_{\tilde{\mathrm{P}}_{i}}\left[\int_{(s, t]} e_{i}^{\top}\left[F^{1}\left(Y_{u-}^{2}, Z_{u}^{2}\right)-F^{2}\left(Y_{u-}^{2}, Z_{u}^{2}\right)\right] \mathrm{d} u \mid \mathcal{F}_{s}\right]=0,
$$

and, therefore, as $e_{i}^{\top}\left[F^{1}\left(Y_{u-}^{2}, Z_{u}^{2}\right)-F^{2}\left(Y_{u-}^{2}, Z_{u}^{2}\right)\right]$ is nonnegative, it must be $0\left(\mathrm{~d} u \times \tilde{\mathrm{P}}_{i}\right)$-a.s. on $[s, t] \times A$. Hence,

$$
\begin{aligned}
0= & e_{i}^{\top}\left[Y_{s}^{1}-Y_{s}^{2}\right]-\mathrm{E}_{\tilde{\mathrm{P}}_{i}}\left[\int_{(s, t]} e_{i}^{\top}\left[F^{1}\left(Y_{u-}^{1}, Z_{u}^{1}\right)-F^{1}\left(Y_{u-}^{2}, Z_{u}^{1}\right)\right] \mathrm{d} u \mid \mathcal{F}_{s}\right] \\
& +\mathrm{E}_{\tilde{\mathrm{P}}_{i}}\left[\int_{(s, t]} e_{i}^{\top}\left[F^{1}\left(Y_{u-}^{2}, Z_{u}^{2}\right)-F^{2}\left(Y_{u-}^{2}, Z_{u}^{2}\right)\right] \mathrm{d} u \mid \mathcal{F}_{s}\right]+e_{i}^{\top} \tilde{X}_{s} \\
= & \mathrm{E}_{\tilde{\mathrm{P}}_{i}}\left[e_{i}^{\top}\left(Q^{1}-Q^{2}\right) \mid \mathcal{F}_{s}\right] .
\end{aligned}
$$

We know that $Q^{1}-Q^{2}$ is nonnegative P-a.s. and, therefore, combining these results for all $i$,

$$
Q^{1}-Q^{2}=0 \quad \text { P-a.s. on } A \text {. }
$$

Finally, we see that, for all $i$ and all $r \in[s, t]$,

$$
\begin{aligned}
0= & \mathrm{E}_{\tilde{\mathrm{P}}_{i}}\left[e_{i}^{\top}\left(Q^{1}-Q^{2}\right) \mid \mathcal{F}_{r}\right] \\
= & e_{i}^{\top}\left[Y_{r}^{1}-Y_{r}^{2}\right]-\mathrm{E}_{\tilde{\mathrm{P}}_{i}}\left[\int_{(r, t]} e_{i}^{\top}\left[F^{1}\left(Y_{u-}^{1}, Z_{u}^{1}\right)-F^{1}\left(Y_{u-}^{2}, Z_{u}^{1}\right)\right] \mathrm{d} u \mid \mathcal{F}_{r}\right] \\
& +\mathrm{E}_{\tilde{\mathrm{P}}_{i}}\left[\int_{(r, t]} e_{i}^{\top}\left[F^{1}\left(Y_{u-}^{2}, Z_{u}^{2}\right)-F^{2}\left(Y_{u-}^{2}, Z_{u}^{2}\right)\right] \mathrm{d} u \mid \mathcal{F}_{r}\right]+e_{i}^{\top} \tilde{X}_{r},
\end{aligned}
$$


and, hence,

$$
e_{i}^{\top}\left[Y_{r}^{1}-Y_{r}^{2}\right]-\mathrm{E}_{\tilde{\mathrm{P}}_{i}}\left[\int_{(r, t]} e_{i}^{\top}\left[F^{1}\left(Y_{u-}^{1}, Z_{u}^{1}\right)-F^{1}\left(Y_{u-}^{2}, Z_{u}^{1}\right)\right] \mathrm{d} u \mid \mathcal{F}_{r}\right]=0 \quad \text { P-a.s. }
$$

By the stronger version of Theorem 1(iv), this proves that, for all $r \in[s, t], Y_{r}^{1}-Y_{r}^{2}=0$, P-a.s. on $A$.

As $Y^{1}-Y^{2}$ is càdlàg, this shows that $Y^{1}=Y^{2}$ on $[s, t] \times A$, up to evanescence.

Remark 6. Equation (8) clearly holds whenever $F$ is monotone decreasing in each component, strictly in at least one component, with respect to $Y$.

Remark 7. Theorem 1 helps distinguish between the understanding of dominance in the classical case and in the nonlinear case generated by BSDEs. In the classical case, no-dominance (or, in a financial setting, 'no-arbitrage') is loosely equivalent to the existence of an equivalent martingale measure for the processes $Y$ (see [12, Section 1.6] for more details). Here, we have assumed the existence of an equivalent supermartingale measure for the processes $e_{i}^{\top} X$. One key difference is that, in the classical linear case, the equivalent martingale measure $\tilde{\mathrm{P}}$ is the same for all terminal values $Q$. In this nonlinear context, Theorem 1(iii) states, in some sense, that there exists an equivalent (super)martingale measure corresponding to each differenced pair of terminal conditions $Q^{1}-Q^{2}$.

\section{Examples}

We here present some examples of applications of these results to situations where the classical results do not apply.

\subsection{A simple single jump time}

First consider the simplest situation, where the only randomness comes from a single random time. We can then obtain concrete conditions for the comparison theorem to hold. These are similar to those in [26], which also includes a Brownian motion. However, our results are obtained through a different approach based on the existence of an equivalent martingale measure.

Let $\tau$ be a random variable taking values in $[0, T]$, the law of which is absolutely continuous with respect to Lebesgue measure. We consider the filtration generated by the indicator process $I_{\{t \geq \tau\}}$. If

$$
H(t)=\mathrm{P}(\tau \in(t, T])=\int_{(t, T]} h_{u} \mathrm{~d} u
$$

where $h$ is the density of $\tau$, then this space posesses a fundamental martingale $M_{t}$ of the form

$$
M_{t}=I_{\{t \geq \tau\}}+\int_{(0, \tau \wedge t]} \frac{\mathrm{d} H_{u}}{H_{u}}=I_{\{t \geq \tau\}}-\int_{(0, \tau \wedge t]} \frac{h}{H_{u}} \mathrm{~d} u,
$$

and there is a martingale representation theorem with respect to $M$ for uniformly integrable martingales. (See [11] for a general theory of stochastic integration in these spaces.) We consider one-dimensional BSDEs with this martingale in the place of $M$ in (2), and $L \equiv 0$. We shall also assume that the driver $F$ is uniformly Lipschitz continuous with respect to $Y$ and, therefore, assumption (iv) of the comparison theorem is trivial, by Theorem 2 . 
Our BSDE will be of the form

$$
\begin{aligned}
Q & =Y_{t}-\int_{(t, T]} F\left(\omega, u, Y_{u-}, Z_{u}\right) \mathrm{d} u+\int_{(t, T]} Z_{u} \mathrm{~d} M_{u} \\
& =Y_{t}-\int_{(t, T]}\left[F\left(\omega, u, Y_{u-}, Z_{u}\right)+\frac{Z_{u} h_{u}}{H_{u}} I_{\{t<\tau\}}\right] \mathrm{d} u+Z_{\tau} I_{\{t \geq \tau\}} .
\end{aligned}
$$

The quantity $X_{r}$ from the comparison theorem is then

$$
\begin{aligned}
X_{r}= & -\int_{(s, r]}\left[F^{1}\left(\omega, u, Y_{u-}^{2}, Z_{u}^{1}\right)-F^{1}\left(\omega, u, Y_{u-}^{2}, Z_{u}^{2}\right)\right] \mathrm{d} u+\int_{(s, r]}\left[Z_{u}^{1}-Z_{u}^{2}\right] \mathrm{d} M_{u} \\
= & -\int_{(s, r]}\left[F^{1}\left(\omega, u, Y_{u-}^{2}, Z_{u}^{1}\right)-F^{1}\left(\omega, u, Y_{u-}^{2}, Z_{u}^{2}\right)+\left(Z_{u}^{1}-Z_{u}^{2}\right) \frac{h_{u}}{H_{u}} I_{\{t<\tau\}}\right] \mathrm{d} u \\
& +\left(Z_{\tau}^{1}-Z_{\tau}^{2}\right) I_{\{t \geq \tau\}} .
\end{aligned}
$$

This has an equivalent supermartingale measure if and only if there exists an equivalent density $\tilde{h}$ for $\tau$ such that

$$
\frac{\tilde{h}_{u}}{\int_{(t, T]} \tilde{h}_{u} \mathrm{~d} u} I_{\{t<\tau\}} \leq \frac{F^{1}\left(\omega, u, Y_{u-}^{2}, Z_{u}^{1}\right)-F^{1}\left(\omega, u, Y_{u-}^{2}, Z_{u}^{2}\right)}{Z_{u}^{1}-Z_{u}^{2}}+\frac{h_{u}}{H_{u-}} I_{\{t<\tau\}} .
$$

Assume that $h>0$. For an equivalent supermartingale measure to exist for all $Y$ and $Z$, positivity of $\tilde{h}$ and changing the role of $Z^{1}$ and $Z^{2}$ shows we require that $F^{1}$ does not vary with $z$ for $t \geq \tau$, that is, for all $y, z^{1}$, and $z^{2}$,

$$
I_{\{t \geq \tau\}} F^{1}\left(\omega, t, y, z^{1}\right)=I_{\{t \geq \tau\}} F^{1}\left(\omega, t, y, z^{2}\right) .
$$

Similarly,

$$
g(u):=\frac{F^{1}\left(\omega, u, Y_{u-}^{2}, Z_{u}^{1}\right)-F^{1}\left(\omega, u, Y_{u-}^{2}, Z_{u}^{2}\right)}{Z_{u}^{1}-Z_{u}^{2}}+\frac{h_{u}}{H_{u}}
$$

must be strictly positive, in which case the function

$$
\tilde{h}_{t}=g(t) \exp \left(-\int_{(0, t]} g(u) \mathrm{d} u\right)
$$

is a candidate for the density, with cumulative distribution function

$$
\int_{(0, t]} \tilde{h}_{t} \mathrm{~d} t=1-\exp \left(-\int_{(0, t]} g(u) \mathrm{d} u\right) .
$$

If $F^{1}$ is Lipschitz continuous with respect to $Z$ then the first term in (9) is bounded. However, as

$$
\frac{h_{t}}{H_{t}}=\frac{h_{t}}{\int_{(t, T]} h_{u} \mathrm{~d} u}=-\frac{\mathrm{d}}{\mathrm{d} t} \log \left(\int_{(t, T]} h_{u} \mathrm{~d} u\right),
$$

and is therefore clearly not integrable on any neighbourhood of $T$, we see that $g$ is not integrable on $(0, T]$. Hence, our candidate density $\tilde{h}$ satisfies

$$
\int_{(0, T]} \tilde{h}_{t} \mathrm{~d} t=1-\exp \left(-\int_{(0, T]} g(u) \mathrm{d} u\right)=1 .
$$


Therefore, $\tilde{h}$ is a valid density on $[0, T]$. Consequently, whenever $g$ is strictly positive, we have constructed an equivalent martingale measure for our process. Clearly, $g$ will be strictly positive whenever

$$
\left|\frac{F^{1}\left(\omega, u, Y_{u-}^{2}, Z_{u}^{1}\right)-F^{1}\left(\omega, u, Y_{u-}^{2}, Z_{u}^{2}\right)}{Z_{u}^{1}-Z_{u}^{2}}\right|<\frac{h_{u}}{H_{u}},
$$

that is, whenever the Lipschitz constant of $F^{1}$ with respect to $Z$ can be bounded above by the (random) process $h_{u} / H_{u}$, the instantaneous rate at which the jump occurs.

\subsection{A general scalar example}

Now consider a pair of general scalar $(K=1)$ BSDEs of the form of (2), with $T$ finite. Assume that $M$ is an $\mathbb{R}^{N}$ martingale with predictable quadratic variation $\langle M\rangle_{t}$ absolutely continuous with respect to $\mathrm{d} t$ and increasing at a rate of at least $\varepsilon \mathrm{d} t$ for some $\varepsilon>0$. All our functions and terminal conditions are, for simplicity, assumed to be bounded. Define $\delta Z=Z^{1}-Z^{2}$ and $\delta_{2} f=F^{1}\left(\omega, u, Y_{u-}^{2}, Z_{u}^{1}\right)-F^{1}\left(\omega, u, Y_{u-}^{2}, Z_{u}^{2}\right)$. Suppose that $F^{1}$ is such that there exists a process $\left(\delta Z^{+}\right)$with values in $\mathbb{R}^{N}$, arranged as a column vector, such that

$$
\left(\delta Z_{t}\right)^{\top}\left(\delta Z_{t}^{+}\right)=1, \quad\left\|\left(\delta_{2} f_{t}\right)\left(\delta Z_{t}^{+}\right)\right\|<C \frac{\mathrm{d}\langle M\rangle_{t}}{\mathrm{~d} t}
$$

for some constant $C$, and

$$
-\frac{\mathrm{d}\langle M\rangle_{t}}{\mathrm{~d} t}<\left(\delta_{2} f_{t}\right)\left(\delta Z_{t}^{+}\right)^{\top}\left(\Delta M_{t}\right) \quad \text { P-a.s. }
$$

Note that $\left(\delta_{2} f_{t}\right)\left(\delta Z_{t}^{+}\right)$can, in some sense, be thought of as a 'derivative' of $F^{1}$. (If $N=1$, this is precisely the ratio considered in (9), and we can see that there the conditions degenerate into those above.) We then define, using the Doléans-Dade exponential,

$$
\Lambda_{t}=1+\int_{(0, t]} \Lambda_{s-}\left(\delta_{2} f_{s}\right) \frac{\mathrm{d} t}{\mathrm{~d}\langle M\rangle_{t}}\left(\delta Z_{s}^{+}\right)^{\top} \mathrm{d} M_{s}
$$

It is easy to verify that, under the stated conditions, $\Lambda$ is a square-integrable martingale, and, hence, defines an equivalent probability measure with $\mathrm{d} \tilde{\mathrm{P}} / \mathrm{dP}=\Lambda_{T}$. Using the general form of Girsanov's theorem [19, p. 169], we see that the process

$$
M_{t}-\int_{(0, t]}\left(\delta_{2} f_{s}\right) \frac{\mathrm{d} s}{\mathrm{~d}\langle M\rangle_{s}}\left(\delta Z_{s}^{+}\right) \mathrm{d}\langle M\rangle_{s}
$$

is a $\tilde{\mathrm{P}}$-local martingale, and, hence, as $\delta L=L^{1}-L^{2}$ is orthogonal to $M$,

$$
\begin{aligned}
X_{t} & =-\int_{(0, t]}\left(\delta_{2} f_{s}\right)\left(\delta Z_{s}\right)\left(\delta Z_{s}^{+}\right) \mathrm{d} s+\int_{(0, t]}\left(\delta Z_{s}\right) \mathrm{d} M_{s}+\delta L_{t} \\
& =-\int_{(0, t]}\left(\delta_{2} f_{s}\right) \mathrm{d} s+\int_{(0, t]}\left(\delta Z_{s}\right) \mathrm{d} M_{s}+\delta L_{t}
\end{aligned}
$$

is a $\tilde{\mathrm{P}}$-local martingale, and, hence, a martingale by boundedness.

Note that this result allows for significant flexibility, due to the possibility of variation in $\left(\delta Z^{+}\right)$. (In some sense, the drift can be allocated to any combination of the components of $M$, so that a new measure can be found under which each component with the associated drift is a martingale.) Furthermore, when $M$ is continuous, the conditions are trivially satisfied. 


\subsection{A vector example with limited comparisons}

We now present a vector example, which demonstrates some of the possibilities in the vector setting. In general, a vector result will only hold in some special cases, but this may nevertheless be of interest.

Consider a general BSDE with two components $(K=2)$, driven by a single Brownian motion $M$. For a vector $z \in \mathbb{R}^{2}$, let $\bar{z}$ and $\underline{z}$ denote the first and second components of $z$. Suppose that $F^{1}$ is of the form

$$
F^{1}(\omega, t, z)=|\bar{z}||\underline{z}| m_{t}
$$

for some bounded process $m: \Omega \times[0, T] \rightarrow \mathbb{R}^{2}$. Consider two BSDEs, with terminal conditions $Q^{1}$ and $Q^{2}$, taking values in $\mathbb{R}^{2}$, where $Q^{2}$ is deterministic. Again, for simplicity, we assume that $Q^{1}$ is bounded. We wish to be able to compare these solutions. This and similar special cases may be of interest in risk management problems, where being able to compare BSDE solutions against known quantities is of use.

We need to be able to verify when the components of $X$ admit equivalent supermartingale measures. Note that $F^{1}$ clearly does not depend on $z$ in a componentwise way, that is, the first component of $F^{1}$ depends on both components of $z$. For our comparison, as $Q^{2}$ is deterministic, the solution satisfies $Y_{t}^{2}=Q^{2}$ and $Z_{t}^{2}=0$ for all $t$.

Consider, without loss of generality, the first component of the solution. We have

$$
\frac{F^{1}\left(\omega, t, Z_{t}^{1}\right)-F^{1}\left(\omega, t, Z_{t}^{2}\right)}{\bar{Z}_{t}^{1}-\bar{Z}_{t}^{2}}=\frac{\left|\bar{Z}_{t}^{1}\right|}{\bar{Z}_{t}^{1}}\left|\underline{Z}_{t}^{1}\right| m_{t} .
$$

We can then again take a measure change using the exponential martingale

$$
\Lambda_{t}=1+\int_{(0, t]} \Lambda_{s-} \frac{\left|\bar{Z}_{t}^{1}\right|}{\bar{Z}_{t}^{1}}\left|\underline{Z}_{t}^{1}\right| \bar{m}_{t} \mathrm{~d} M_{t} .
$$

Under the measure thus defined, Girsanov's theorem states that the first element of $X$ is a local martingale, and, hence, a martingale by boundedness. A similar result holds for the second component. Hence, we can make a componentwise comparison between the solutions of our BSDE and any deterministic terminal condition.

\subsection{A vector example with rotation}

We now consider a final vector example, where the solution to our BSDE depends only on the component of $Z$ lying on a given line. This example could also be considered using rotation and a componentwise comparison theorem. We shall here consider it directly.

Consider a general BSDE with two components $(K=2)$, driven by a single Brownian motion $M$. For a vector $z \in \mathbb{R}^{2}$, let $\bar{z}$ and $\underline{z}$ denote the first and second components of $z$. Suppose that we have a driver of the form

$$
F(\omega, t, y, z)=f(\omega, t, \bar{z}-\underline{z})\left[\begin{array}{c}
1 \\
-1
\end{array}\right]
$$

for some bounded function $f: \Omega \times[0, T] \times \mathbb{R}^{2 \times 1} \rightarrow \mathbb{R}$, Lipschitz continuous with respect to $\bar{z}-\underline{z}$, with $f(\omega, t, 0)=0$. Now consider the subspace $\mathcal{Q}$ of the set of terminal conditions such that, for any $Q \in \mathcal{Q}, \bar{Q}=-\underline{Q}$, that is, the first component is the negative of the second. We shall show that a comparison theorem holds between elements of $\mathcal{Q}$. Note, however, that a comparison need not hold between terminal conditions not in $\mathcal{Q}$. 
We note that, for any $Q \in \mathcal{Q}$, as $f$ does not depend on $y$, the solution to the BSDE must satisfy $\bar{Z}=-\underline{Z}$. Hence, in this subspace, the solutions are equal to those for

$$
F(\omega, t, y, z)=f(\omega, t, 2 \bar{z})\left[\begin{array}{c}
1 \\
-1
\end{array}\right]=f(\omega, t,-2 \underline{z})\left[\begin{array}{c}
1 \\
-1
\end{array}\right] .
$$

The existence of an equivalent martingale measure for the each component is then a standard application of Girsanov's theorem.

\section{Applications to nonlinear expectations}

A useful consequence of these comparison properties is that they allow us to develop a theory of nonlinear expectations, in the same way as Peng [25]. These are closely related to the theory of dynamic risk measures, as in [1], [3], [27], and others, as each concave nonlinear expectation $\mathcal{E}\left(\cdot \mid \mathcal{F}_{t}\right)$ corresponds to a dynamic convex risk measure through the relationship

$$
\rho_{t}(Q)=-\mathcal{E}\left(Q \mid \mathcal{F}_{t}\right) .
$$

A further discussion of this relationship can be found in [27]. (In a similar way to [21], [22], and others, we here write $\rho_{t}(Q)=-\mathcal{E}\left(Q \mid \mathcal{F}_{t}\right)$ rather than $\rho_{t}(Q)=\mathcal{E}\left(-Q \mid \mathcal{F}_{t}\right)$, as this draws a closer conceptual connection between $\rho$ as a convex risk functional, and $\mathcal{E}$ as a concave utility functional.)

For simplicity, we shall, for the remainder of this paper, assume that the drivers $F$ considered are such that

$$
\mathbb{Q}_{s, t}^{F}=\left\{Q \in L^{0}\left(\mathcal{F}_{t}\right): \mathrm{E}\left[\|Q\|^{2} \mid \mathcal{F}_{s}\right]<+\infty, \mathrm{P} \text {-a.s. }\right\} .
$$

That is, for any $\mathcal{F}_{t}$ measurable random variable with $\mathrm{P}$-a.s. finite $\mathcal{F}_{s}$ conditional variance, there exists a unique solution to the BSDE (2) on $[s, t]$, satisfying certain conditions, such as square integrability, which we shall leave as implicit. For simplicity, this set will be denoted $L_{s}^{2}\left(\mathcal{F}_{t}\right)$. For consistency, this requires that, for all $r \in[s, t]$, the solution $Y_{r}$ to (2) satisfies $Y_{r} \in \mathbb{Q}_{s, r}^{F}=L_{s}^{2}\left(\mathcal{F}_{r}\right)$. Note that $L^{0}\left(\mathcal{F}_{r}\right) \subseteq L_{r}^{2}\left(\mathcal{F}_{t}\right) \subseteq L_{s}^{2}\left(\mathcal{F}_{t}\right)$ for all $r \leq s \leq t$.

As in [6], we make the following generalisation of a definition of [25].

Definition 2. For $s \leq t \leq T$, fix the sets $\mathcal{Q}_{s, t} \subseteq L_{s}^{2}\left(\mathcal{F}_{t}\right)$. A system of operators

$$
\mathcal{E}_{s, t}: L_{s}^{2}\left(\mathcal{F}_{t}\right) \rightarrow L^{0}\left(\mathcal{F}_{s}\right), \quad 0 \leq s \leq t \leq T,
$$

is called an $\mathcal{F}$-consistent nonlinear evaluation for $\left\{\mathcal{Q}_{s, t}\right\}$ defined on $[0, T]$ if $\mathcal{E}_{s, t}$ satisfies the following properties.

1. For $Q, Q^{\prime} \in \mathcal{Q}_{s, t}$, if $Q \geq Q^{\prime}$, P-a.s. componentwise then

$$
\varepsilon_{s, t}(Q) \geq \varepsilon_{s, t}\left(Q^{\prime}\right) \quad \mathrm{P} \text {-a.s. }
$$

componentwise, with equality if and only if $Q=Q^{\prime}$, P-a.s.

2. For $Q \in L^{0}\left(\mathcal{F}_{t}\right), \varepsilon_{t, t}(Q)=Q$, P-a.s.

3. For any $r \leq s \leq t$ and any $Q \in L_{r}^{2}\left(\mathcal{F}_{t}\right)$,

$$
\mathcal{E}_{s, t}(Q) \in L_{r}^{2}\left(\mathscr{F}_{S}\right)
$$

and

$$
\mathcal{E}_{r, s}\left(\mathcal{E}_{s, t}(Q)\right)=\mathcal{E}_{r, s}(Q) \quad \mathrm{P} \text {-a.s. }
$$

4. For any $s \leq t, A \in \mathcal{F}_{s}$, and $Q \in L_{s}^{2}\left(\mathcal{F}_{t}\right), I_{A} \varepsilon_{s, t}(Q)=I_{A} \varepsilon_{s, t}\left(I_{A} Q\right)$, P -a.s. 
Definition 3. Fix a driver $F$ and time points $s \leq t$. For any $Q^{1}, Q^{2} \in \mathbb{Q}_{s, t}^{F}=L_{s}^{2}\left(\mathcal{F}_{t}\right)$, let $\left(Y^{1}, Z^{1}\right)$ and $\left(Y^{2}, Z^{2}\right)$ be the associated solutions of the BSDE (2).

Suppose that, for some set $\mathcal{Q}_{s, t} \subseteq L_{s}^{2}\left(\mathcal{F}_{t}\right)$ and all $Q^{1}, Q^{2} \in \mathcal{Q}_{s, t}$,

1. for each $i$, there exists a measure $\tilde{\mathrm{P}}_{i}$ equivalent to $\mathrm{P}$ such that the $i$ th component of $X$, as defined for $r \in[s, t]$ by

$$
\begin{aligned}
e_{i}^{\top} X_{r}:= & -\int_{(s, r]} e_{i}^{\top}\left[F\left(\omega, u, Y_{u-}^{2}, Z_{u}^{1}\right)-F\left(\omega, u, Y_{u-}^{2}, Z_{u}^{2}\right)\right] \mathrm{d} u \\
& +\int_{(s, r]} e_{i}^{\top}\left[Z_{u}^{1}-Z_{u}^{2}\right] \mathrm{d} M_{u}+e_{i}^{\top}\left[L_{r}^{1}-L_{r}^{2}\right],
\end{aligned}
$$

is a $\tilde{\mathrm{P}}_{i}$-supermartingale on $[s, t]$,

2. $F$ is componentwise Lipschitz continuous in $Y$, in the sense of Remark 5. (This includes the case when $F$ does not depend on $Y$.)

Then $F$ will be called balanced on $\mathcal{Q}_{s, t}$. Additionally, $F$ will be called balanced on a family $\left\{\mathcal{Q}_{s, t}\right\}$ if it is balanced on each member of the family.

Remark 8. The distinction between the sets $\mathcal{Q}_{s, t}$ and $L_{s}^{2}\left(\mathcal{F}_{t}\right)$ is that $L_{s}^{2}\left(\mathcal{F}_{t}\right)$ is the set on which solutions to the BSDE exist, whereas $\mathcal{Q}_{s, t}$ is the set on which a (strict) comparison theorem holds. In many cases, these sets may be identical. However, we distinguish between them for the sake of generality.

Remark 9. Again, it is possible to modify Definition 3 in the same way as in Corollary 1.

Theorem 5. Fix a driver $F$ balanced on some family $\left\{\mathcal{Q}_{s, t}\right\}$. Define the 'F-evaluation', $a$ system of operators on $L_{s}^{2}\left(\mathcal{F}_{t}\right)$ for all $s<t$, by

$$
\mathcal{E}_{s, t}(Q)=Y_{s},
$$

where $Y_{s}$ is the solution to the BSDE (2). Then $\mathcal{E}_{s, t}$ is an $\mathcal{F}_{t}$-consistent nonlinear evaluation for $\left\{Q_{s, t}\right\}$.

Proof. We verify that conditions 1-4 of Definition 2 are satisfied.

1. The statement $\varepsilon_{s, t}\left(Q_{1}\right) \geq \varepsilon_{s, t}\left(Q_{2}\right)$, P-a.s. whenever $Q_{1} \geq Q_{2}$, P-a.s. is simply the result of the strict comparison theorem (Theorem 1 with Theorems 2 and 3 and Remark 5), which holds as $F$ is balanced on $\mathcal{Q}_{s, t}$.

2. The fact that $\varepsilon_{t, t}(Q)=Q$, P-a.s. for any $Q \in L^{0}\left(\mathscr{F}_{t}\right)$ is trivial, as we have defined $\varepsilon_{t, t}(Q)$ by the solution to a BSDE, which reaches its terminal value $Q$ at time $t$ by construction.

3. To show that $\varepsilon_{r, s}\left(\mathcal{E}_{s, t}(Q)\right)=\mathcal{E}_{r, t}(Q)$, P-a.s. for any $r \leq s \leq t$, let $Y$ denote the solution to the relevant BSDE. Then a simple rearrangement of (2) gives

$$
Y_{s}=Y_{r}-\int_{(r, s]} F\left(\omega, u, Y_{u-}, Z_{u}\right) \mathrm{d} u+\int_{(r, s]} Z_{u}^{\top} \mathrm{d} M_{u}+\int_{(r, s]} \mathrm{d} L_{u} .
$$

Hence, $Y_{r}$ is also the time $r$ value of a solution to the BSDE with terminal time $s$ and value $Y_{s}$. Therefore, it is clear that $Y_{s} \in L_{r}^{2}\left(\mathcal{F}_{S}\right)$, and, by the uniqueness of BSDE solutions,

$$
\varepsilon_{r, s}\left(\mathcal{E}_{s, t}(Q)\right)=\mathcal{E}_{r, t}(Q) \quad \mathrm{P} \text {-a.s., }
$$

as desired. 
4. We wish to show that, for $A \in \mathcal{F}_{s}, I_{A} \varepsilon_{s, t}(Q)=I_{A} \varepsilon_{s, t}\left(I_{A} Q\right)$, P-a.s. For $Q \in L_{s}^{2}\left(\mathcal{F}_{t}\right)$, let $Y^{1}$ be the solution to the BSDE (2) with terminal condition $Q$. Premultiplying by $I_{A}$, we have a BSDE with terminal condition $I_{A} Q$, driver $I_{A} F$, and solution $I_{A} Y_{s}^{1}=I_{A} \varepsilon_{s, t}(Q)$. If $Y^{2}$ is the solution to the BSDE with terminal condition $I_{A} Q$ then we similarly obtain $I_{A} Y_{s}^{2}=I_{A} \varepsilon_{s, t}\left(I_{A} Q\right)$. We can now write

$$
Y_{u}^{3}=I_{A} Y_{u}^{2}+I_{A^{\mathrm{c}}} Y_{u}^{1}
$$

for $u \in[s, t]$. It follows that

$$
\begin{aligned}
Y_{s}^{3}- & \int_{(s, t]}\left[I_{A} F\left(\omega, t, Y_{u}^{3}, Z_{u}^{3}\right)+I_{A^{\mathrm{c}}} F\left(\omega, t, Y_{u}^{3}, Z_{u}^{3}\right)\right] \mathrm{d} u+\int_{(s, t]} Z_{u}^{3} \mathrm{~d} M_{u} \\
& =I_{A} Q+I_{A^{\mathrm{c}}} Q
\end{aligned}
$$

which clearly implies that $Y^{1}$ and $Y^{3}$ are both solutions at $s$ to the BSDE with driver $F$, terminal condition $Q \in L_{S}^{2}\left(\mathcal{F}_{S}\right)$, and, hence, $Y^{1}=Y^{3}$ up to indistinguishability. Therefore, $I_{A} Y_{S}^{2}=I_{A} Y_{S}^{1}$, P-a.s., as desired.

With a slight modification, we also define nonlinear expectations.

Definition 4. For $t \leq T$, fix the sets $\mathcal{Q}_{t} \subseteq L_{t}^{2}\left(\mathcal{F}_{T}\right)$. A system of operators

$$
\mathcal{E}\left(\cdot \mid \mathcal{F}_{t}\right): L_{t}^{2}\left(\mathcal{F}_{T}\right) \rightarrow L^{0}\left(\mathcal{F}_{t}\right), \quad 0 \leq t \leq T,
$$

is called an $\mathscr{F}_{t}$-consistent nonlinear expectation for $\left\{\mathcal{Q}_{t}\right\}$ defined on $[0, T]$ if

- the restriction $\mathcal{E}_{s, t}=\left.\mathscr{E}\left(\cdot \mid \mathcal{F}_{S}\right)\right|_{L_{s}^{2}\left(\mathcal{F}_{t}\right)}$ of $\mathscr{E}$ to $L_{s}^{2}\left(\mathcal{F}_{t}\right)$ is a nonlinear evaluation for all $s \leq t$,

- for all $t$, and any $A \in \mathcal{F}_{t}, Q \in L_{t}^{2}\left(\mathcal{F}_{T}\right)$,

$$
I_{A} \mathcal{E}\left(Q \mid \mathcal{F}_{t}\right)=\mathcal{E}\left(I_{A} Q \mid \mathcal{F}_{t}\right) \quad \text { P -a.s. }
$$

Theorem 6. Consider a driver $F$ balanced on a family $\left\{\mathcal{Q}_{s, t}\right\}$ with

$$
F\left(\omega, u, Y_{u_{-}}, 0\right)=0 \quad(\mathrm{~d} u \times \mathrm{P})-\text { a.s. }
$$

on $[0, T] \times \Omega$.

For each $Q \in L_{S}^{2}\left(\mathcal{F}_{T}\right)$, define

$$
\mathcal{E}\left(Q \mid \mathcal{F}_{s}\right):=Y_{s},
$$

where $Y_{S}$ is the solution to (2) with $t=T$.

Then $\mathcal{E}\left(\cdot \mid \mathcal{F}_{S}\right)$ is a nonlinear expectation on $\left\{\mathcal{Q}_{S}:=\mathcal{Q}_{s, T}\right\}$. In this case it will also be called an $F$-expectation.

Proof. From Theorem 5, we can see that the restriction of this operator is a nonlinear evaluation.

We know that $I_{A} F\left(\omega, t, Y_{t-}, Z_{t}\right)=F\left(\omega, t, I_{A} Y_{t-}, I_{A} Z_{t}\right),(\mathrm{d} t \times \mathrm{P})$-a.s. and $I_{A} Q \in L_{t}^{2}\left(\mathcal{F}_{T}\right)$. Therefore, if $(Y, Z, L)$ is the unique solution to the BSDE with driver $F$ and terminal value $Q$, then we can premultiply the BSDE (2) by $I_{A}$ to see that $\left(I_{A} Y, I_{A} Z, I_{A} L\right)$ is the unique solution to the BSDE with driver $F$ and terminal value $I_{A} Q$. That is, $I_{A} \mathcal{E}\left(Q \mid \mathcal{F}_{t}\right)=I_{A} Y_{t}=$ $\mathcal{E}\left(I_{A} Q \mid \mathcal{F}_{t}\right)$. 


\subsection{Geometry of $\boldsymbol{F}$-evaluations}

The comparison theorem establishes various geometric properties of the BSDE solutions, or, equivalently, of the $F$-evaluations. Some of these properties are explored in this section.

Theorem 7. Suppose that $\mathcal{Q}_{s, t}$ is a convex set, with $F$ a balanced driver on $\mathcal{Q}_{s, t}$. Suppose that $F$ is concave on $\mathcal{Q}_{s, t}$, that is, for any $\lambda \in[0,1]$ and any $\left(Y^{1}, Z^{1}\right),\left(Y^{2}, Z^{2}\right)$ corresponding to $Q^{1}, Q^{2} \in \mathcal{Q}_{s, t}$, $(\mathrm{d} t \times \mathrm{P})$-a.s. on $[s, t]$,

$$
\begin{aligned}
& F\left(\omega, t, \lambda Y_{t-}^{1}+(1-\lambda) Y_{t-}^{2}, \lambda Z_{t}^{1}+(1-\lambda) Z_{t}^{2}\right) \\
& \quad \geq \lambda F\left(\omega, t, Y_{t-}^{1}, Z_{t}\right)+(1-\lambda) F\left(\omega, t, Y_{t-}^{2}, Z_{t}^{2}\right),
\end{aligned}
$$

the inequality being taken componentwise.

Then, for any $\lambda \in[0,1]$ and any $Q^{1}, Q^{2} \in \mathcal{Q}_{s, t}$, the $F$-evaluation is strictly concave, that is, it satisfies

$$
\mathcal{E}_{s, t}\left(\lambda Q^{1}+(1-\lambda) Q^{2}\right) \geq \lambda \varepsilon_{t, T}\left(Q^{1}\right)+(1-\lambda) \mathcal{E}_{s, t}\left(Q^{2}\right),
$$

with equality if and only if $Q^{1}=Q^{2}$, P-a.s.

Proof. Taking a convex combination of the BSDEs with terminal conditions $Q^{1}$ and $Q^{2}$ gives the equation

$$
\begin{aligned}
& \lambda Y_{s}^{1}+(1-\lambda) Y_{s}^{2}-\int_{(s, t]}\left[\lambda F\left(\omega, u, Y_{u-}^{1}, Z_{u}\right)+(1-\lambda) F\left(\omega, u, Y_{u-}^{2}, Z_{u}^{2}\right)\right] \mathrm{d} u \\
& +\int_{(s, t]}\left[\lambda Z_{u}^{1}+(1-\lambda) Z_{u}^{2}\right] \mathrm{d} M_{u} \\
& \quad=\lambda Q^{1}+(1-\lambda) Q^{2},
\end{aligned}
$$

which is a BSDE with terminal condition $\lambda Q^{1}+(1-\lambda) Q^{2}$ and driver

$$
\tilde{F}=\lambda F\left(\omega, u, Y_{u-}^{1}, Z_{u}\right)+(1-\lambda) F\left(\omega, u, Y_{u-}^{2}, Z_{u}^{2}\right) .
$$

Consider the BSDE with terminal condition $\lambda Q^{1}+(1-\lambda) Q^{2}$ and driver $F$. Denote the solution to this by $Z^{\lambda}$. We can compare these BSDEs using Theorem 1 . The assumptions are all satisfied as $F$ is balanced on $\mathcal{Q}_{s, t}$. Hence, the solutions satisfy

$$
Y^{\lambda} \geq \lambda Y^{1}+(1-\lambda) Y^{2}
$$

By the strict comparison, which holds as $F$ is balanced, we have equality if and only if the terminal conditions are equal with conditional probability 1 . The result follows.

Theorem 8. Consider a driver $F$ balanced on a family $\left\{\mathcal{Q}_{s, t}\right\}$ (which may be empty). Suppose that, for some $s<t$ and all deterministic $\lambda$ in some set $\mathcal{C}$ where all products are assumed to be well defined,

$$
F\left(\omega, u, \lambda Y_{u-}, \lambda Z_{u}\right)=\lambda F\left(\omega, u, Y_{u-}, Z_{u}\right) \quad(\mathrm{d} u \times \mathrm{P})-a . s .
$$

on $[s, t] \times \Omega$. Then the nonlinear evaluation generated by $F$ satisfies

$$
\varepsilon_{s, t}(\lambda Q)=\lambda \varepsilon_{s, t}(Q)
$$

for all $Q \in L_{s}^{2}\left(\mathcal{F}_{t}\right)$ and all $\lambda \in \mathcal{C}$. 
Proof. Simply take the BSDE with terminal condition $Q$, premultiply by $\lambda$, and factor the driver $F$ term. It is clear that this is then the BSDE with terminal condition $\lambda Q$, and that the solution is $(\lambda Y, \lambda Z, \lambda L)$.

Remark 10. Note that this theorem applies for both scalar and square matrix valued $\lambda$, and it is clear that, with appropriate modifications to allow for dimensionality of $F$, would apply for vector-valued $\lambda$ as well.

Definition 5. For any time $t$, we define $H_{t}(Q)$, the essential convex hull of $Q$ at time $t$, to be the smallest, $\widetilde{F}_{t}$-measurable, convex set such that $\mathrm{P}\left(Q \in H_{t}(Q) \mid \widetilde{F}_{t}\right)=1$.

Definition 6. We denote by $H_{t}^{\mathrm{ri}}(Q)$ the relative interior of $H_{t}(Q)$, that is, the interior of $H_{t}(Q)$ viewed as a subset of the affine hull it generates.

Remark 11. The interested reader is referred to any good book on elementary stochastic finance for a more detailed definition, for example, [15, p. 65] or [17, p. 27].

Theorem 9. Consider a nonlinear F-expectation $\mathcal{E}\left(\cdot \mid \mathcal{F}_{t}\right)$ for $\left\{\mathcal{Q}_{t}\right\}$, with

$$
L^{0}\left(\mathcal{F}_{t}\right) \subseteq Q_{t}
$$

for all $t$. Then, for all $Q \in \mathcal{Q}_{t}$, each component $e_{i}^{\top} \mathcal{E}\left(Q \mid \mathcal{F}_{t}\right)$ satisfies

$$
e_{i}^{\top} \mathcal{E}\left(Q \mid \mathcal{F}_{t}\right) \in H_{t}^{\mathrm{ri}}\left(e_{i}^{\top} Q\right)
$$

Proof. For a fixed $t$, define a random variable $Q^{\text {min }}$ by

$$
e_{i}^{\top} Q^{\min }=\inf H_{t}\left(e_{i}^{\top} Q\right) .
$$

Note that $Q>Q^{\text {min }}$ componentwise. As $Q^{\min }$ is $\mathcal{F}_{t}$-measurable, the solution to the BSDE with driver $F$ and terminal condition $Q^{\min }$ is simply $Y_{s}=Q^{\min }$ for $s \geq t$. As $Q^{\min } \in L^{0}\left(\mathcal{F}_{t}\right) \subseteq Q_{t}$, property 1 of Definition 2 implies that either $H_{t}(Q)$ contains only a single point, in which case

$$
Q=Q^{\min } \in H_{t}(Q)=H_{t}^{\mathrm{ri}}(Q),
$$

or

$$
\mathcal{E}\left(Q \mid \mathcal{F}_{t}\right)>Q^{\min }
$$

componentwise. We can repeat this argument with $Q^{\max }$ defined by $e_{i}^{\top} Q^{\max }=\sup H_{t}\left(e_{i}^{\top} Q\right)$, which shows that either $H_{t}(Q)$ contains a single point, or $\mathscr{E}\left(Q \mid \mathcal{F}_{t}\right)<Q^{\text {max }}$ componentwise. Hence, in the latter case, $e_{i}^{\top} \mathcal{E}\left(Q \mid \mathcal{F}_{t}\right)$ lies strictly within the interior of $H_{t}\left(e_{i}^{\top} Q\right)$, which is the same as $H_{t}^{\mathrm{ri}}\left(e_{i}^{\top} Q\right)$. In either case this shows that

$$
e_{i}^{\top} \mathcal{E}\left(Q \mid \mathcal{F}_{t}\right) \in H_{t}^{\mathrm{ri}}\left(e_{i}^{\top} Q\right)
$$

\section{Conclusion}

In this paper we have presented a comparison theorem for backward stochastic differential equations (BSDEs) in which the stochastic term is given by an arbitrary martingale. This result is a generalisation of the result of Peng [24], as it allows for martingales other than Brownian motion, and also applies to the case of vector-valued equations. We have shown how, under some conditions, for example, Lipschitz continuity, the conditions of this theorem can be simplified. 
We have defined the concept of a balanced driver for a BSDE, which is essentially a condition on the driver such that a comparison theorem holds. By expressing this condition in terms of equivalent (super)martingale measures, the links with previous work on arbitrage theory are more apparent.

Using these results, we have developed a theory of nonlinear expectations, which can now lie in a general probability space. These are closely related to dynamic risk measures, as emphasised in [27]. Various applications of this theory are possible, as we have not assumed that the martingale $M$ used to define the BSDEs will generate the filtration of the probability space. We have also outlined some general geometric properties of these nonlinear expectations.

\section{Acknowledgement}

Robert Elliott wishes to thank the Australian Research Council for support.

\section{References}

[1] Artzner, P. et al. (2007). Coherent multiperiod risk adjusted values and Bellman's principle. Ann. Operat. Res. 152, 5-22.

[2] Barles, G., Buckdahn, R. And Pardoux, E. (1997). Backward stochastic differential equations and integralpartial differential equations. Stoch. Stoch. Reports 60, 57-83.

[3] Barrieu, P. and El Karoui, N. (2004). Optimal derivatives design under dynamic risk measures. In Mathematics of Finance (Contemp. Math. 351), eds G. Yin and Q. Zhang, American Mathematical Society, Providence, RI, pp. 13-25.

[4] Cheredito, P. and Stadje, M. (2010). BS $\Delta$ Es and BSDEs with non-Lipschitz drivers: comparison, convergence and robustness. Preprint. Available at http://arxiv.org/abs/1002.0175v1.

[5] Cohen, S. N. and Elliott, R. J. (2008). Solutions of backward stochastic differential equations on Markov chains. Commun. Stoch. Anal. 2, 251-262.

[6] Cohen, S. N. And Elliott, R. J. (2010). A general theory of finite state backward stochastic difference equations. Stoch. Process. Appl. 120, 442-466.

[7] Cohen, S. N. And Elliott, R. J. (2010). Backward stochastic difference equations and nearly-time-consistent nonlinear expectations. In preparation.

[8] Cohen, S. N. And Elliott, R. J. (2010). Comparisons for backward stochastic differential equations on Markov chains and related no-arbitrage conditions. Ann. Appl. Prob. 20, 267-311.

[9] Cohen, S. N. AND Elliott, R. J. (2010). Existence and comparisons for BSDEs in general spaces. In preparation.

[10] Coquet, F., Hu, Y., Memin, J. and Peng, S. (2002). Filtration-consistent nonlinear expectations and related g-expectations. Prob. Theory Relat. Fields 123, 1-27.

[11] Davis, M. H. A. (1976). The representation of martingales of jump processes. SIAM J. Control Optimization 14, 623-638.

[12] Delbaen, F. and Schachermayer, W. (2006). The Mathematics of Arbitrage. Springer, Berlin.

[13] El Karoui, N. And Mazliak, L. (eds) (1997). Backward Stochastic Differential Equations. Pitman, London.

[14] Elliott, R. J. (1982). Stochastic Calculus and Applications. Springer, New York.

[15] Elliott, R. J. ANd Kopp, P. E. (2005). Mathematics of Financial Markets, 2nd edn. Springer, New York.

[16] El Otmani, M. (2008). Backward stochastic differential equations associated with Lévy processes and partial integro-differential equations. Commun. Stoch. Anal. 2, 277-288.

[17] Föllmer, H. And Schied, A. (2002). Stochastic Finance (de Gruyter Stud. Math 27). Walter de Gruyter, Berlin.

[18] Hu, Y. And Peng, S. (2006). On the comparison theorem for multidimensional BSDEs. C. R. Acad. Sci. Paris 343, 135-140.

[19] Jacod, J. And Shiryaev, A. N. (2003). Limit theorems for Stochastic Processes, 2nd edn. Springer, Berlin.

[20] JIA, G. (2009). Some uniqueness results for one-dimensional BSDEs with uniformly continuous coefficients. Stat. Prob. Lett. 79, 436-441.

[21] Jobert, A. And Rogers, L. C. G. (2008). Valuations and dynamic convex risk measures. Math. Finance 18, $1-22$.

[22] Klöppel, S. AND Schweizer, M. (2007). Dynamic indifference valuation via convex risk measures. Math. Finance 17, 599-627.

[23] Lin, Q. Q. (2003). Nonlinear Doob-Meyer decomposition with jumps. Acta Math. Sinica 19, 69-78.

[24] Peng, S. G. (1992). A generalized dynamic programming principle and Hamilton-Jacobi-Bellman equation. Stoch. Stoch. Reports 38, 119-134. 
[25] Peng, S.-G. (2005). Dynamically consistent nonlinear evaluations and expectations. Preprint. Available at http://arxiv.org/abs/math/0501415.

[26] Peng, S. And Xu, X. (2009). BSDEs with random default time and their applications to default risk. Preprint. Available at http://arxiv.org/abs/0910.2091v1.

[27] Rosazza Gianin, E. (2006). Risk measures via g-expectations. Insurance Math. Econom. 39, 19-34.

[28] Royer, M. (2006). Backward stochastic differential equations with jumps and related non-linear expectations. Stoch. Process. Appl. 116, 1358-1376.

[29] Situ, R. (2005). Theory of Stochastic Differential Equations with Jumps and Applications. Springer, New York.

[30] ZHOU, Q. (2007). On comparison theorem and solutions of BSDEs for Lévy processes. Acta Math. Appl. Sinica 23, 513-522. 